\title{
ECONOMÍA POLÍTICA DEL REGIONALISMO - CASO ANGOLA Y NAMIBIA.
}

\author{
Ezequiel Israel Jonas ${ }^{1}$ \\ https://orcid.org/0000-0002-8961-6535
}

\section{RESUMEN}

Esta investigación tiene como objetivo desarrollar una revisión teórica sobre el regionalismo en el marco de la economía política, según diversas contextualizaciones que se han planteado sobre éste; tomando el caso de Angola y Namibia como expresión de este proceso en el continente africano. Al respecto, se brinda una conceptualización acerca del tema, ofrecida por autores como Sánchez Ortiz (2009), Murillo Zamora (2004), Bernal Meza (2009), quienes lo definen como un proceso dirigido a alcanzar el desarrollo endógeno, la conformación de grandes espacios económicos, el incremento y la diversificación de la producción en países africanos, específicamente en Angola y Namibia. Para recabar la información se procedió con la revisión, lectura, análisis e interpretación de información recabada de distintas fuentes documentales, organizadas atendiendo a los temas abordados en este artículo. Como conclusiones destacan que la regionalización pretende favorecer el desarrollo económico, social, político y cultural de los países africanos, dentro de los cuales en Angola y Namibia se ha llevado a cabo un proceso de integración dirigido a conformar una red de acuerdos, cuya administración ha estado signada por la presencia de dificultades que han limitado el logro de los objetivos trazados, así como en cuanto al cumplimiento y desarrollo de acuerdos bilaterales de cooperación.

Palabras clave: regionalismo; regionalización; integración económica; Angola; Namibia.

\section{Economia Política Do Regionalismo - Caso Angola E Namíbia \\ RESUMO}

Esta investigação visa desenvolver uma revisão teórica do regionalismo no quadro da economia política, de acordo com várias contextualizações que foram levantadas sobre o mesmo; tomando o caso de Angola e da Namíbia como uma expressão deste processo no continente africano. A este respeito, fornece uma conceptualização da questão, oferecida por autores como Sánchez Ortiz (2009), Murillo Zamora (2004), Bernal Meza (2009), que a definem como um processo destinado a alcançar o desenvolvimento endógeno, a formação de grandes espaços económicos, o aumento e diversificação da produção nos países africanos, especificamente em Angola e Namíbia. A fim de recolher a informação, procedemos à revisão, leitura, análise e interpretação da informação recolhida de diferentes fontes documentais, organizada de acordo com as questões abordadas neste artigo. Como conclusões, salientam que a regionalização visa favorecer o desenvolvimento económico, social, político e cultural dos países africanos, dentro dos quais em Angola e na Namíbia foi levado a cabo um processo de integração com vista à formação de uma rede de acordos, cuja administração tem sido marcada pela presença de dificuldades que têm limitado a realização dos objectivos estabelecidos, bem como em termos de cumprimento e desenvolvimento dos acordos de cooperação bilateral.

Palavras-chave: regionalismo; regionalização; integração económica; Angola; Namíbia.

\section{Political Economy of Regionalism - Angola And Namibia Case}

\section{ABSTRACT}

This research aims to develop a theoretical review of regionalism in the framework of political economy, according to various contextualizations that have been raised about it, taking the case of Angola and Namibia as an expression of this process in the African continent. In this regard, it provides a conceptualization of the issue, offered by authors such as Sánchez Ortiz (2009), Murillo Zamora (2004), Bernal Meza (2009), who define it as a process aimed at achieving endogenous development, the formation of large economic spaces, the increase and diversification of production in African countries, specifically in Angola and Namibia. To gather the information, we proceeded to review, read, analyze and interpret the information gathered from different documentary sources, organized according to the topics addressed in this article. As conclusions, it should be pointed out that regionalization aims at favoring the economic, social, political and cultural development of African countries, within which in Angola and

\footnotetext{
${ }^{1}$ Centro de Estudos Internacionais-ISCTE-IUL, Portugal. Email: Ezequiel Jonas@iscte-iul.pt
} 
Namibia an integration process has been carried out aimed at forming a network of agreements, whose administration has been marked by the presence of difficulties that have limited the achievement of the objectives set, as well as in terms of the compliance and development of bilateral cooperation agreements.

Keywords: regionalism; regionalization; economic integration; Angola; Namibia.

\section{Introducción}

El regionalismo, a consideración de Rodríguez Suárezy Ochoa (2014), bajo el enfoque de una perspectiva teórica general, sugiere el antagonismo de la autarquía ${ }^{2}$ y del unilateralismo, a causa de la consideración de la inclusión entre sus más elevadas prioridades, del fomento de la cooperación entre las regiones, en menoscabo de las acciones particulares y unilaterales de las grandes potencias. De una manera más específica, Rodríguez Suárez y Ochoa (2014, p. 81) definen el regionalismo desde la perspectiva de la cooperación entre diferentes naciones, las cuales establecen unconjunto de alianzas en la región donde pertenecen. Como complemento de este significado, el regionalismo se vincula conceptualmente a una política de Estado, o incluso, de la sociedad civil, o bien, de los actores protagónicos de las relaciones internacionales, cuyo propósito es la promoción e incremento de la cooperación interregional.

Asimismo, la regionalización esconcebida como un proceso complementario, o al menos paralelo, asociado al de globalización, cuya contraparte se expresa por medio de un fenómeno político denominado regionalismo o integración regional, la cual surge como una oportunidad para el enfrentamiento de los problemas globales, posibilitando además, un nivel superior de formulación y coordinación de propósitos comunes entre grandes áreas (Pipitone, 1996).

En la actualidad, resulta prioritaria para un considerable número de países de la comunidad internacional ${ }^{3}$ la búsqueda de emergentes modelos de cooperación, enfocados desde perspectivas cuantitativas y cualitativas; y particularmente, desde la visión de la cooperación interregional. En efecto, cabe mencionar que las integraciones regionales se fundamentan en los intereses compartidos y en un sentido de pertenencia; de hecho, los pilares para el establecimiento de regionalismos se cimientan en beneficios comunes, con semejanza en los sistemas políticos y económicos, que se complementan económicamente, con afinidad cultural, tradición, y en algunos casos, hasta en una religión en común (Rodríguez Suárez y Ochoa, 2014, p. 81).

En aras de la comprensión de la naturaleza de los regionalismos del siglo XXI, un elemento útil para ello, es la consideración de que los intereses económicos tienen una mayor influencia que los políticos; bajo estas premisas, las ventajas de la cooperación interregional se encaminan en los momentos actuales, hacia el aprovechamiento de los beneficios propios de la proximidad geográfica y los mercados regionales; de igual modo, en el aprovechamiento de las ventajas competitivas de las economías de la región, lo cual, a mediano y a largo plazo,aportarápaz, estabilidad y prosperidad a la zona involucrada.

En este orden de ideas, se planteó una investigación con el objetivo de desarrollar una revisión teórica del regionalismo en el marco de la economía política, dadas sus diferentes contextualizaciones; en este caso, la representada por Angola y Namibia como expresión del regionalismo en el continente africano; así, hubo que realizar un arqueo de fuentes documentales de corte científico, versados sobre la temática seleccionada; a tal efecto, fueron consultados loscuarenta (40) documentos, de mayor confiabilidad, actualidad, pertinencia y correspondencia con el tema, contentivos de libros, artículos arbitrados, entre otros; su información fue resumida, registrada para ser organizada según los tópicos abordados. Posterior, se redactó la versión del presente artículo.

\section{Regionalismo, regionalización e integración}

\footnotetext{
2En este contexto, el término “autarquía” hace referencia a una política de un Estado que procura bastarse con sus propios recursos (Real Academia Española, 2018). ${ }^{3} \mathrm{El}$ concepto de "comunidad internacional" ha sido ampliamente debatido en la política internacional.

Éste se encuentra referido a la representación, hipotética o virtual, de una moralidad universal la cual pudiera estar compartida mayoritariamente por los gobiernos y ciudadanos de todo el mundo. Así, se requiere de dos postulados desde el momento en que se recurre a la existencia de tal entidad moral: uno gira en relación con la existencia de una moralidad internacional impuesta a la política internacional y, otra, vinculada con que la opinión mayoritaria es necesariamente superior, legítima o moralmente justa (Diccionario del Poder Mundial, 2018). 
Indistintamente, los conceptos de "regionalismo" y "regionalización" son empleados como sinónimos de integración económica, bajo la perspectiva del marco de la economía política, porlo cual sugiere la necesidad de precisarlos, en vista de que el regionalismo involucra dimensiones más amplias; ellas no siempre están asociadas con la integración económica, con la política y relaciones exteriores. Bernal Meza (2009, p. 3) sugiere que estas dos expresiones parecieran referirse al mismo proceso propio de la economía global del momento que hoy se vive; unida a la concepción de que se intenta identificar una serie de dimensiones de la mundialización como proceso.

Asimismo, el concepto y el contenido de la "región" son esenciales para comprender el estudio del regionalismo (Grant y Söderbaum, 2003; Hettne y Söderbaum, 2000). A consideración de Sepúlveda Ramírez(2001), la "región" se concibe, política y económicamente, una categoría analítica. Las Ciencias Sociales se han encargado de brindar distintos aportes referidas a su evolución teórica (Alfred Weber, Losch y Christaller, Perroux, Friedman y Weaver, Markussen y Gore, Scout y Storpe, Krugman y Obstfeld, Masahisa, Krugman y Venables; citados por Mellado, 2013) ${ }^{4}$. No obstante, la actual configuración espacial de las actividades productivas y comerciales, conlleva a la reorganización del espacio que puede ser interpretado como un "producto social", compuesto por la configuración territorial y la dinámica social.

La configuración territorial, se refiere a una serie de componentes de tipo natural que han sido objeto de modificación con la intervención de la mano del hombre; así como gracias a la dinámica de las relaciones sociales entre diferentes conglomerados humanos, en un espacio y en un tiempo dado (Santos, 1996, p. 62). Consecuentemente, la ocupación y los usos de los territorios han sufrido una notoria transformación, tanto físico, como en lo histórico-cultural, con la finalidad de reposicionarse espacial competitivamente (Mellado, 2013). Así entonces, el término "región" se interpreta como un espacio para la integración política, económica y social (Haesbaert, 2005). En términos idealistas, Macridis y Hulliung (1996, citados por Massó, 2009, p. 107) abordan el concepto de región de la siguiente forma:

De esta forma, se interpreta la región como un espacio geográfico determinado, compuesto por un conjunto de estados unidos por prácticas, creencias comunes, distintas formas de comunicación, económicas y hasta personales; en ellas suele existir un apego a los valores regionales, en los cuales se sustenta la cooperación y la búsqueda de la paz y la convivencia entre naciones-estado individuales; se asume que tales valores y arreglos institucionales podrían reemplazar las entidades nacionales, leales y con instituciones que las representen.

Es importante considerar el hecho, la integración regional es estimulada para fortalecer la identidad, la unidad y la preservación de la paz. Bajo estas premisas, la región pudiera ser entendida como una respuesta a la necesidad de reorganización de comunidades políticas para evitar tensiones entre estados (Oyarzún, 2008; citada por Amado, 2018, p. 51).

En aras de diferenciar claramente los términos de "regionalización" y "regionalismo", Murillo (2004) señala que el primero, es el proceso que facilita la conformación de regiones o espacios económicos; por su parte, el regionalismo se corresponde con la disposición explícita de definir acuerdos e instituciones regionales. Así, la regionalización puede ser concebida como un espacio donde se desarrolla el regionalismo, y su manifestación es la integración ${ }^{5}$.

Parafraseando a Bernal Meza (2009; p. 67-70), la regionalización, desde el punto de vista político, implica crear lealtad entre distintos estados autónomos en su política interna y externa, para ampliar sus esquemas de regulación social y económica; ejemplo de ello es la Unión Europea, así, hay una configuración de la economía política mundial que representa una fuerza de transformación del sistema social en el mundo.

\footnotetext{
${ }^{4}$ Son numerosas las discusiones vinculadas al concepto región. El término ha sido objeto de diversas interpretaciones por parte economistas, geógrafos, científicos sociales o historiadores, quienes lo emplean para hacer referencia a un área de actividad productiva (ejemplo, "región agrícola") o un área geográfica, ejemplo un Estado (Amado, 2018).

${ }^{5}$ Expertos en política internacional han sometido a discusión las bondades del regionalismo y del universalismo como un componente dirigido a solidificar la paz del mundo. Los defensores de este enfoque, suelen coincidir en que la integración regional es el punto intermedio entre la integración mundial y la anarquía internacional; asimismo, asumen que la afinidad económica, cultural, política y geográfica en la región propicia una organización más efectiva (Couloumbis y Wolfe, 1979; p. 361; citados por Bernal, 2009). 
En el contexto de las relaciones internacionales, el regionalismo es definido como una emergente formación regional con su propia dinámica, es decir, un subsistema supranacional dentro del sistema internacional (Pereira, 2008). No se refiere solamente a territorios geográficamente cercanos, con políticas exteriores interrelacionadas (Cantori y Spiegel, 1970; citados por Sainz, 2008) ni tampoco está condicionado a indicadores aislados e independientes uno del otro, como la proximidad geográfica, (cuyo ejemplo es la relación de Israel con sus vecinos o Rusia con los países de la Unión Europea, entre otros ), la interdependencia económica (como por ejemplo el caso de la relación comercialentre los países de China y Angola) y la homogeneidad, (que supone el compartir un sistema económico, religión, niveles de desarrollo, idioma, historia en común y otros aspectos similares) (Amado, 2018).

Para Sánchez Ortiz (2009, p. s/n), el regionalismo es un mecanismo que viene a responder a las necesidades de la globalización, atendiendo a criterios como la proximidad geográfica y al flujo comercial que se produce entre varias naciones de una región, el abaratamiento del transporte y la posibilidad de oportuna entrega de los productos y servicios a mercados de gran consumo.

Para Bernal Meza (2009), el regionalismo se refiere específicamente a tres aspectos no necesariamente coincidentes, pero posiblemente compatibles. El primero, abarca la perspectiva de las relaciones internacionales, bajo la cual, el regionalismo involucra la creación, expansión o integración de, o a un subsistema. Un segundo aspecto, se asocia a la óptica de la política exterior; donde el regionalismo puede referirse a un paradigma referido a una política externa de estados nacionales. Por último, en el contexto de la economía política, regionalismo implica niveles de integración tanto de tipo económica como comercial en un área geográfica que forma parte de una región; así, regionalismo es entendido como integración.

Ahora bien, esta integración económica es el proceso de creciente asociación de las economías de países dirigidas a disminuir obstáculos en los intercambios comerciales para incrementar sus beneficios (Sánchez, 2009).No obstante, todo el proceso de integración regional depende de la evolución económica y política de los países miembros (Mistry, 2003; citado por Söderbaum y Shaw, 2003).

Considerando las preferencias y protecciones de la integración, la dimensión conceptual del regionalismo, es expresada a través de su contraposición a la globalización, o también llamada "mundialización" de la economía política mundial; por medio de la cual, se estarían derribando las barreras que restringen o dificultan o la circulación de bienes y servicios a escala planetaria. Partiendo de esa perspectiva y bajo el supuesto de que la mundialización involucra ciertas amenazas para los países y sus sociedades, sobre todo las empobrecidas económica y políticamente, a criterio de Bernal Meza (2009), la integración sería conveniente por las siguientes razones:

a. Colocar límites la degradación o erosión a la que pudiera estar sometido un Estado, a través de su capacidad de regulación, referida a la facultad de otorgar ymovilizar recursos, instituir las áreas de mayor importancia en el desarrollo sectorial yprotegerlas condiciones para que haya un crecimiento sumado al desarrollo.

b. Maximizar el proceso de recuperación de acumulación de capitales nacionales (privados y públicos) como una estrategia de desarrollo.

c. Fortalecer el rol del sector privado nacional, dado su poder hegemónico dentro del Estadonación; para transformarse en un actor modernizador, dinámico y cambiante de la estructura socio-económica.

d. Contrarrestar las tendencias objetivas y circunstancias estructurales negativas de la mundialización.

\section{Economía política del regionalismo - Del regionalismo a la integración económica}

El regionalismo, según lo afirmado por Mata Diz y Volpini Silva (2011), debe ser planteado como la etapa inicial que precede a la integración regional, desde el punto de vista económico o político. En términos generales, el regionalismo se dirige a eliminar las barrerasentrelos países, mientras que 
los procesos de integración suponen la intensificación de las relaciones entre éstos, permitiendo el alcance de un grado de identidad que supera el mero establecimiento de pactos inter-fronterizos. De acuerdo a lo circunscrito en el encuadre organizacional políticode un Estado, desde la visión del Derecho Internacional clásico, el regionalismo no trae consigo la transformación interna o de homogenización por parte de los Estados. Es posible entonces, deducir una principal distinción entre el regionalismo y la integración, la cual justamente hace referencia a las consecuencias que ambos generan, especialmente en relación a la modificación de la estructura del Estado nacional. Por otra parte, la regionalización y el regionalismo se conciben como procesos interestatales cuyo fin es detener el progreso del mercado sin fronteras y globalizado; por tanto, se instituye como una alternativa para las naciones signatarias de acuerdos de cooperación o integración para adquirir una presencia mayor en el contexto mundial, esencialmente relacionada con la competencia en el mercado internacional de los productos.

No obstante, atendiendo a lo propuesto por Hurrell (1995; citado por Oyarzún, 2008), la integración regional es uno de los distintos procesos vinculados con el regionalismo y una alternativa beneficiosa para que los países se inserten de manera competitiva en el mercado mundial. Por otra parte, es pertinente tener en cuenta la multiplicidad de factores que suceden al proceso de integración, entre los que destacan como principales: la gestión nacional (en vista de que se requiere la planificación estratégicapara gestionar organizadamente); donde los actores que protagonizan el procesosean los agentes privados o civiles y las instituciones para garantizar laintegración regional, para propiciar la generación de recursos humanos con el talento necesario (Amado, 2018).

Efectivamente, puede interpretarse que la integración representa un mecanismo que ha facilitado la alianza entre sectores económicos de distintas naciones para estimular una de-reglamentación y la eliminación de políticas en economías que requieren reformas para lograr un mayor desarrollo. Así entonces, según Amado (2018) son variados los estudios que se han dedicado a analizarla influencia de la integración regional sobre las economías de las naciones involucradas, la unificación de sus Estados miembros, asícomola cesión de competencias de estos a los órganos comunes.

Resulta evidente entonces, que la economía de mercado ha traído como consecuencia un aumento de la interdependencia económica y social, así como con la difusión mundial de sus ideologías. La movilidad de capitales, bienes, servicios y de las personas han sido promovidas por la eliminación de obstáculos previos a la globalización y a los acuerdos de cooperación e integración. Por ende, la integración se entiende como un tipo de regionalismo que le otorga a la región la facultad de lograr convertirse en un importante actor del sistema, donde se realizan intercambios comerciales, haycooperación económica y sociopolítica, más allá de las relaciones intergubernamentales, vinculada con diferentes actores componentes del Estado(militares, trabajadores, empresarios, diplomáticos,parlamentarios, estudiantes, ambientalistas, etcétera) hacia una entidad supranacional.

Asimismo, en ocasiones, ejercen gran influencia actores no estatales o privados, incluso más destacados en las relaciones internacionales que los mismos. Debido a esto, debe considerarse que cada vez más, los diferentes actores, sean ellos estatales o privados, emergen e influyen, internacionalmente, en las tomas de decisiones. Por otra parte, con la globalización se propició el incremento de la interdependencia entre los Estados, así como también el auge de los conflictos; ya sean de carácter local o no, éstos se han generalizado al extremo de afectar en forma directa a los intereses regionales y expandirse hasta alcanzar dimensiones internacionales.

Pareciera obvio que la integración regional viene a formar parte de una estructura organizacional novedosa para los Estados, en la cual, se da el surgimiento a relaciones internas y externas novedosas comunes, el espacio integrado. En esencia, ha habido un cambio en la noción externa e interna sobre el concepto de Estado, para conceptualizarse en un escenario de ampliación de las fronteras territoriales (Fernández, 1992). En general, los supuestos que se asocian al avance de la integración dependerán enormemente del fin planteado por los países involucrados en función de 
la búsqueda de intereses comunes; en el caso de objetivos estrictamente económicos, por ello, se pudiera decir que es un proceso de cooperación. Al respecto, Murillo (2004) añade que la integración es una gradual construcción de agentes nuevos, fiel expresión de su identidad e intereses comunes de sus miembros, comprende una transferencia de responsabilidades, deberes y potestadesde una gente a otra en una región determinada, todo con el fin de alcanzar sus fines, según los intereses de cada parte involucrada, en ello se constatan avances y retrocesos, por ello su carácter dinámico, condicionado por factores tanto internos como externos.

La integración regional es una herramienta estratégicamente efectiva que debe ser aplicada de forma continua para afrontar los retos impuestos por la globalización (Benavides de La Vega, 2011); es parcial dados los flujos comerciales y de capital dentro de un escenario internacional, con una gran influencia sobre el crecimiento económico de las naciones que se han integrado.

\section{La integración regional en África}

En África se ha experimentado una serie de transformaciones culturales, ampliamente influenciada por la introducción, en la colonia, de hábitos de culturas extranjeras; cuando, se intentó unificar en un mismo territorio administrativo a diferentes pueblos con distintas características sociales, políticas e ideológicas con la consecuente creación de nuevas instituciones que terminaron acentuando las discrepancias y el aislamiento regional de las relaciones entre las naciones, que incluso, manejaban lenguas diferentes. Posteriormente al proceso independentista, se buscó subsanar los problemas africanos que habían ocasionado la separación de pueblos y la agrupación de otros totalmente distintos que anteriormente ocupaban Estados propios.

África es un continente con una notable y enorme diversidad étnica, cultural y política vinculada a unas condiciones de pobreza extrema ${ }^{6}$; productode la deficiente gestión de sus líderes o de la desigualdistribuciónde los recursos; y en otros, de la marcada presencia de constantes conflictos entre los pueblos por las diferencias sociales internas, muchas de las cuales fueron heredadas de la época colonial. Paradójicamente, esta realidad contrasta con la gran cantidad de riquezas naturales que concentra el continente, entre las que destacan: la plata ( $79 \%$ de las reservas mundiales), fosfato, uranio, cobre, petróleo, diamantes y recursos hídricos; además de la existencia de suelos peculiarmente ricos en minerales y muy propicios para el pasto. Además, ha tenido un crecimiento económico que supera la tasa del 5\% durante los dos últimos años (Amado, 2018).

Es un continente conformado por cincuenta y cinco (55) naciones; ilógica y arbitrariamente sus fronteras actuales han sido modificadas y delimitadas; la expansión imperial europea ha irrespetado los límites geográficos, naturales y étnicos antiguo. Asimismo, los acontecimientos históricos del hombre y sus sociedades han contribuido con su división en África del Norte o del Sahara y el Sur del Sahara (el África Negra). No obstante, el continente africano no ha permanecido al margen del fenómeno globalizado del regionalismo; pues, hasta ahora ha participado en la firma de acuerdos que han contribuido en la conformación de distintosbloques de integración de tipo económico, para solventar los problemas que acontecen en muchos de los países de este continente (Bartesaghi y Pereira, 2015).

Para Benavides (2011), la integración en África podría sugerir, una ampliación del espacio económico, así como la creación de infraestructuras que favorezcan la interdependencia del continente, permitiendo la viable superación de los problemas propios de la fragmentación económica y geográfica para propiciar la calidad de vida de los africanos.

De hecho, esta integración económica inicialmente se constituyó en un instrumento que formó parte de la estrategia fundamental para alcanzar la estabilidad y el desarrollo de los países de la región, con posterioridad a su independencia de los regímenes coloniales. No obstante, para entender este proceso debe considerarse el hecho de que el continente africano está caracterizado por una serie de naciones divididas, con economías limitadas y poco estables; por tanto, la conformación de espaciosde amplitud mayor puedan favorecer el desarrollo de economías de más

\footnotetext{
${ }^{6} \mathrm{La}$ fragmentación geográfica es una características notoria en África, zona donde se localizan las naciones del mundo más empobrecidas económica, educativamente, en infraestructura, así como en materia sanitaria (Bartesaghi y Pereira, 2015). 
amplia escala, ha sido uno de los factores que ha impulsado la aplicación de variados esquemas de integración, el cual fue un modelo que adicionalmente se vislumbraba como una alternativa viable frente al fracaso de las políticas nacionales de una porción significativa de los países del continente (Kabunda Badi, 2009). En complemento, la constitución de 53 estados, en gran parte de pequeña extensión, ha hecho de la integración una estrategia ineludible y atractiva; la misma se ve fortalecida por dos procesos del momento, a saber, la globalización y la mundialización organizada en la conformación de bloques en las diferentes regiones, en el caso africano, para atender a sus necesidades de desarrollo.

Una característica destacada en el mundo delaintegración, es el nivel de compromiso que ha sido asumido en relación a la conformación de bloques. A nivel internacional, las zonas y tratados de libre comercio son los mecanismos mayormente empleados para lograrlo; sin embargo, en África se desplegó la conformación de uniones aduaneras, herramienta ésta que ha resultado inflexible por su incapacidad de adaptación a la velocidad de los cambiantes escenarios a nivel internacional, generando efectos obstaculizadores para éxito de los procesos de integración, a los que se suma la presencia de una perjudicial densidad institucional representada por la conformación de parlamentos y por la existencia de Tribunales de Justicia.

A consideración de Bartesaghi y Pereira (2015; p.10), la masiva articulación de instituciones vinculadas a la integración, no garantiza el logro de los objetivos planteados en los tratados internacionales o en el cumplimiento de las normas establecidas, lo que sucede con frecuencia es que no hay una estructura institucional apropiada en países y regiones con inestabilidad política. Por otra parte, resalta también en el contexto de la integración africana, una superposición de procesos; con la consecuente participación de varios países en más de una unión aduanera.

Tabla 1. Procesos de integración africana.

\begin{tabular}{|l|l|l|l|}
\hline Proceso & Origen & Ubicación & $\begin{array}{l}\text { Cantidad de } \\
\text { miembros }\end{array}$ \\
\hline $\begin{array}{l}\text { Unión del Madreb } \\
\text { Árabe (UMA) }\end{array}$ & 1989 & África del Norte & 5 \\
\hline $\begin{array}{l}\text { Mercado Común para } \\
\text { el este y el Sur de África } \\
\text { (COMESA) }\end{array}$ & 1994 & $\begin{array}{l}\text { África del Sur y del } \\
\text { Centro }\end{array}$ & 19 \\
\hline $\begin{array}{l}\text { Comunidad Económica } \\
\text { de los Estados del } \\
\text { África Central (ECCAS } \\
\text { - CEEAC) }\end{array}$ & 1983 & $\begin{array}{l}\text { África del Sur y del } \\
\text { Centro }\end{array}$ & 10 \\
\hline $\begin{array}{l}\text { Comunidad Económica } \\
\text { de los Estados del } \\
\text { África Occidental } \\
\text { (ECOWAS - } \\
\text { CEDEAO) }\end{array}$ & 1975 & África del oeste & 15 \\
\hline $\begin{array}{l}\text { Comunidad de } \\
\text { Desarrollo de África } \\
\text { Austral (SADC) }\end{array}$ & 1992 & África del Sur y del & 15 \\
\hline $\begin{array}{l}\text { Comunidad del África } \\
\text { Oriental (EAC) }\end{array}$ & 1999 & Este & 5 \\
\hline $\begin{array}{l}\text { Unión Económica y } \\
\text { Monetaria de África } \\
\text { Occidental (UEMOA) }\end{array}$ & 2003 & África del Este & 5 \\
\hline $\begin{array}{l}\text { Comunidad Económica } \\
\text { y Monetaria de África } \\
\text { Central (CEMAC) }\end{array}$ & 1994 & África del Oeste & 8 \\
\hline $\begin{array}{l}\text { Unión Aduanera de } \\
\text { África Austral (SACU) }\end{array}$ & 1910 & África del Sur & 5 \\
\hline
\end{tabular}


Fuente: Bartesaghi y Pereira (2015).

En la actualidad, África cuenta con más de 200 organizaciones regionales, más que cualquier otro continente, donde cada nación se integra a más de una de estas experiencias o iniciativas de cooperación en sectores bancarios, financieros, aduaneros, agrícolas o ambientales, así como las uniones políticas con una cierta cesión de soberanía. Según el Gobierno de España (2018), dentro de las agrupaciones regionales en el continente, de mayor significación, se mencionan de seguidas:

- Unión Africana (UA): ha sido creado en el año 2002, esta organización supranacional,es la sucesora de la Organización para la Unidad Africana (OUA), cuya fundación data de 1963, la integran todos los países del continente, al reincorporarse Marruecos, en el año 2017. Los objetivos primarios se orientan a la conquista de la cohesión, unidad y solidaridad entre las naciones africanas, la defensa de la soberanía, la integridad territorial e independencia de sus participantes, en otras palabras, fortalecer la integración continental, garantizar la estabilidad democrática, la seguridad y la paz.

- Comunidad Económica y Monetaria de África Central (CEMAC): fue constituida en el año 1994, para integrar económicamente a aquellas naciones que emplean el franco CFA como moneda; dentro de los países que la integran se encuentran Chad, República del Congo, República Centroafricana, Camerún,Guinea Ecuatorial y Gabón.

- Comunidad Económica de los Estados de África Central (CEEAC): fue institucionalizada en 1983, para estimular el desarrollo social, cultural y económico, la paz y seguridad de países como Camerún, Chad, República Centroafricana, Guinea Ecuatorial, República del Congo, Gabón, Angola, República Democrática del Congo, Burundi, Sao Tomé y Príncipe.

- Comunidad Económica de los Estados de los Grandes Lagos (CEPGL): su establecimiento data del año 1976, la conforman Burundi, la República Democrática del Congo y Ruanda. Cuenta como objetivo promover la integración económica y la seguridad regional entre sus Estados miembros.

- Conferencia Internacional de la Región de los Grandes Lagos (CIRGL). Su conformación data del año 2000, la integran naciones como Ruanda, Burundi, la República Democrática del Congo, República Centro Africana, Angola,Sudán, Sudán del Sur, Uganda, Tanzania,Zambia, Kenia y República del Congo. Desarrolla acciones para garantizar la democracia, el desarrollo económico, humanitario de los países que la componen.

- Mercado Común para África del Este y el Sur (COMESA): es una organización que ha sido fundada en 1994, esta unión aduanera agrupa a Burundi, República Democrática del Congo, Comores, Yibuti, Eritrea, Egipto, Etiopía, Libia, Kenia, Madagascar, Mauricio, Ruanda, Malaui, Seychelles, Sudán, Uganda, Suazilandia, Zambia y Zimbabue.

- Comunidad Económica del África del Oeste (CEDEAO): Fue creada en 1975, está compuesta por naciones como Burkina Faso,Benín, Cabo Verde, Gambia, Costa de Marfil,Ghana, Guinea Bissau, Guinea,Malí,Liberia, Níger, Senegal, Nigeria,Sierra Leona y Togo. Su radio de actuación se orienta a tres áreas, a saber: la cooperación económica regional, el comercio y la integración económica, a través de la realización de proyectos comunitarios.

- Comunidad para el Desarrollo del África Austral (SADC): está integrada por Angola, República Democrática del Congo, Botswana, Lesoto, Mauricio, Malaui, Mozambique, Suazilandia, Namibia, Tanzania, Zimbabue, Zambia, Seychelles, Sudáfrica y Madagascar (miembro suspendido debido al golpe de Estado de 2009). Su fecha de creación data del año 1979, el propósito que persigue es alcanzar el crecimiento y desarrollo económico sostenible y equitativo,mediante la implementación de sistemas eficientes de producción, ayuda a los sectores menosfavorecidos para superar la pobreza; así mismo,aspira a la integración y cooperación y la garantía de la seguridad y la paz (Bach, 1998).

- Autoridad Intergubernamental para el Desarrollo (IGAD): Fue creada en1996, es la organizaciónsucesoradela Autoridad intergubernamental para la Sequía y el Desarrollo, que 
había sido creada previamente una década atrás. Se encuentra compuesta porKenia,Etiopía, Somalia, Uganda,Sudán, Eritrea, Yibuti y Sudán del Sur. Fue reorganizada y se le incluyó nuevas áreas de cooperación regional; cuenta como misión coordinar la ayuda de sus Estados miembros para garantizar la seguridad alimentaria, la protección del medio ambiente, la promoción y el mantenimiento de la seguridad y la paz, la integración y cooperación económicas. Actúa como mediador en elconflicto que presenta Sudán del Sur desde diciembre de 2013.

- Banco Africano de Desarrollo (BAfD): Está compuesta por 55 naciones africanas y 27 de países no africanos, su creación data de 1964. Su objetivo principal es la lucha contra la pobreza, por medio de la estimulación del crecimiento y progreso social y económico sostenible. Dentro de sus áreas prioritarias de acción están el desarrollo de infraestructuras, integración económica regional, con la participación y desarrollo del sector privado, la formación técnica y profesional y la gobernanza económica.

A pesar de la gran cantidad de intentos por solidificar un proceso de integración regional exitoso en el continente africano, son muchos los desafíos que deben ser enfrentados para que ésta sea una herramienta que efectivamente promueva el desarrollo económico; entre las principales barreras, el Banco de Desarrollo Africano (citado por Bartesaghi y Pereira, 2015) resume las siguientes:

- Múltiples membrecíasde los Estados en diversos procesos de integración ${ }^{7}$.

- Escaza o nula credibilidad en las instituciones.

- Falta de compromiso por los países participantes.

- Inexistente armonización de políticas regionales y nacionales.

No obstante, ante dicho escenario, Mballa (2008) afirma que los obstáculos a la integración africana son cuantiosos, pero no insuperables. Para ello, la reconstrucción multidimensional de su entorno deberá tener en cuenta la reconsideración del potencial de su pasado, así como la participación del pueblo en cada proyecto de desarrollo constructivo; lo cual implica una mirada hacia la historia, la idiosincrasia y la cultura de los pueblos en todo el territorio. La complejidad de la realidad endógena del continente plantea nuevos y profundos horizontes de análisis y estudio.

Asimismo, Kabunda Badi(2009) señala que la integración regional en África es factible únicamente cuando haya una superación de los egoísmos de las distintas naciones y puedan coordinar proyectos industriales a nivel de cada país y en el escenario continental para responder a los intereses del desarrollo equilibrado, de la infraestructura y del transporte que contribuya con el comercio interafricano y se pueda lograr la paz, mediante la disminución de los conflictos.

\section{El caso de Angola y Namibia}

Dick Lord (2008) y Steenkamp (2006) refieren con respecto a la frontera entre Angola y Namibia, ambas ubicadas en el oeste del África Austral, se puede conseguir del lado namibio a las ciudades conocidas con el nombre de Rundu, Eenhana, Ruacana, Nkurenkuru y Oshikango.Al este de Rundu, se localizan decenas de kilómetros el río Okavango, hasta formar el delta del Okavango en Botswana. Por el lado oeste, está el curso del río Kunene hasta el océano Atlántico, atravesando las cataratas Epupa. Es Kunene el foco de diversos proyectos de cooperación entre ambos países, dentro de los cuales se incluyenproyectos hidroeléctricos, de acopio transfronterizo en agua, entreotros.

\footnotetext{
${ }^{7}$ La participación en más de un bloque simultáneamente, implica que las economías tienen que realizar aportes financieros a diferentes procesos, lo que afecta aquellas economías poco estables de los países africanos; por ello, se constituye en un problema que se le suma ala coexistencia de diferentes normas técnicas y regulatorias (no armonizadas entre sí) que limitan el desarrollo integral de la zona. Esta duplicación de esfuerzos y recursos constituyen limitaciones para la integración económica, sobre todo cuando se producen conflictos de intereses y de normas entre los bloques (AFDB, 2011; citado por Bartesaghi y Pereira, 2015) 


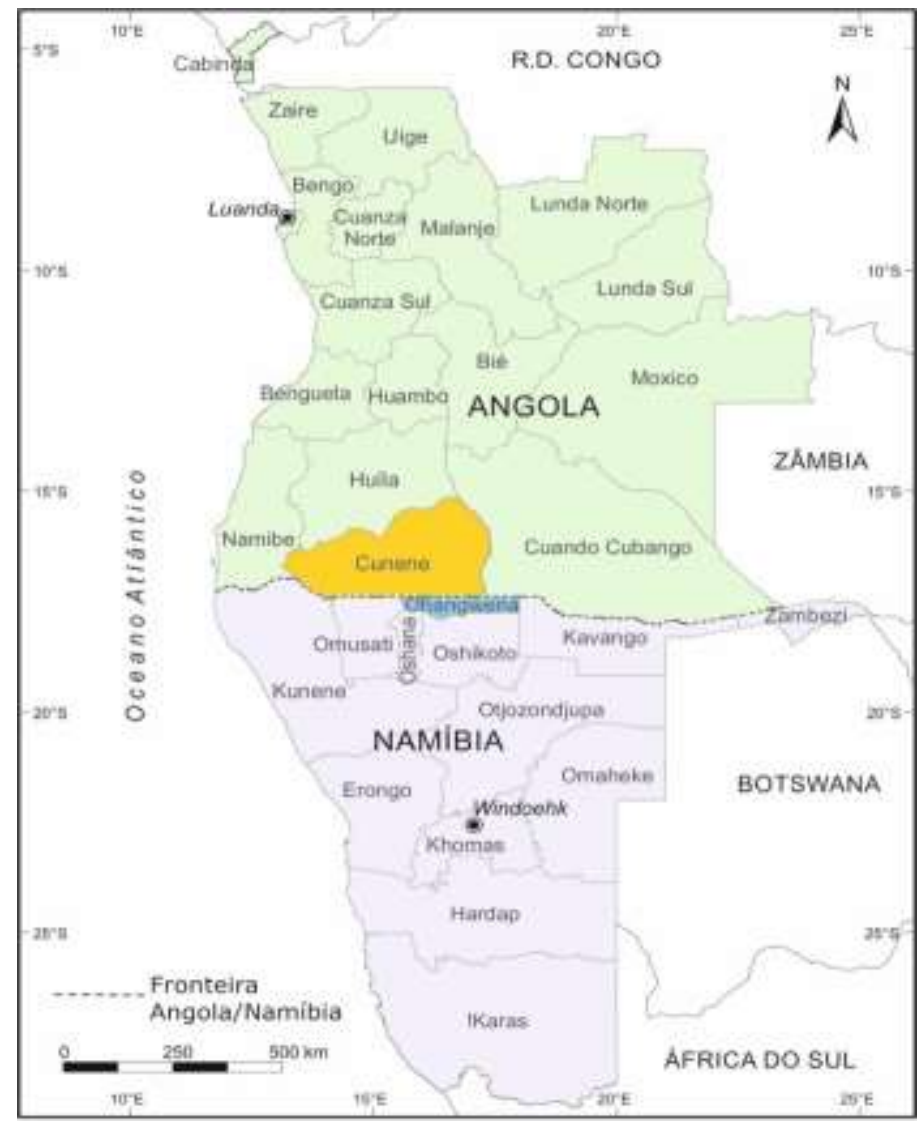

Figura 1: Frontera entre Angola y Namibia.

Fuente: Google Map.

Las relaciones entre Angola y Namibia se remontan históricamente al momento en el cual Namibia logra su independencia con la realización del movimiento de la Organización de los Pueblos del Sudoeste Africano (SWAPO), ella fue una larga batalla en Angola, luego de la II Guerra Mundial y posterior a la independencia ocurrió en Cuito Cuanavale, región situada al sudeste de Angola, en la provincia de Kuando-Kubango; entre 15 de noviembre de 1987 y 23 de marzo de 1988, fue el escenario de grandes enfrentamientos entre las fuerzas armadas de Sudáfrica del apartheid, Angola y el ejército cubano, terminando con la derrota definitiva de los sudafricanos, dando origen a la independencia de Namibia y poniendo fin al régimen del apartheid en Sudáfrica. El proceso de independencia de Angola sirvió de apoyo a las operaciones guerrilleras de la nación vecina, conocida como África del Suroeste, para alcanzar su independencia de Sudáfrica, hechos acontecidos en 1990 y que dio lugar a su nuevo nombre de Namibia (Iliffe, 1998).

Efectivamente, la relación política entre Namibia y Angola se hizo muy intensa durante el conflicto angoleño. Los esfuerzos de pacificación de Namibia en Angola subrayaron su creencia de que establecer la paz era necesariopara alcanzar la seguridad regional de la SADC. No obstante, para cualquier comunidad regional, el establecimiento de la paz es una necesidad, un bien común y un ingrediente para promover, mantener y garantizar la seguridad en dicha comunidad (Hamutenya, 2014). En los procesos de integración africanos, Angola y Namibia son países de participación común en la Unión Africana (UA), en la Comunidad para el Desarrollo del África Austral (SADC), en el Banco Africano de Desarrollo (BAfD), entre los más importantes.

Podría afirmarse que Angola y Namibia han arraigado lazos culturales, políticos, sociales, económicos e históricos que datan de hace muchos años. Según Hamutenya (2014), Angola es un Estado geográficamente estratégico a Namibia, y viceversa. Los partidos políticos en el poder en ambos países son vistos como aliadosexpertos que, en un momento dado, compartieron una plataforma ideológica y un enfoque de sus respectivas luchas de liberación. Con Namibia, 
alcanzando finalmente su independencia en el año de 1990, ambos Estados establecieron representaciones diplomáticas y mejoraron las relaciones mutuas.

Tras emerger de décadas de guerra, Angola es en la actualidad el mercado más grande para cualquier comercio en el sur del continente africano, gracias a sus programas de reconstrucción en diversos ámbitos; lo cual ofrece un abanico ilimitado de posibilidades a Namibia para exportación. Para el año 2014, Angola representaba el diez por ciento (10\%) de las exportaciones totales de Namibia; aunque esta cifra ha ido en incremento en la medida que se abren más oportunidades y los dos gobiernos mejoran las áreas de cooperación bilateral (Hamutenya, 2014).

Por otra parte, es pertinente mencionar que los acuerdos vigentes entre Angola y Namibia incluyen áreas asociadas al comercio: Acuerdo bilateral sobre comercio y economía (Hamutenya, 2014) y Acuerdo bilateral sobre Promoción recíproca y protección de inversiones (Hamutenya, 2014); a la salud: ambos países son parte de la iniciativa subregional para eliminar la malaria e integran el Trans-Kunene (TKMI) como una alianza estratégica entre Namibia y Angola para trabajar en el control de la malaria a lo largo de su frontera común; y a la prevención del delito: a través de la Comisión Conjunta de Defensa y Seguridad Angola-Namibia, la cual discute asuntos que conciernen a la seguridad, la libre circulación de personas en la frontera, especialmente en lo que se refiere a los residentes fronterizos.

Angola también se registró en el año 2014, como el tercer mayor destino de exportación para Namibia después de Sudáfrica y el Reino Unido. Adicionalmente, Angola y Namibia son miembros comunes de organizaciones regionales e internacionales, donde además de las ya mencionadas, destaca la Organización Mundial del Comercio (OMC); lo cual demuestra su visión compartida de trabajar juntos para alcanzar metas comunes a nivel de plataformas regionales y mundiales. Dichos objetivos incluyen a nivel regional, el desarrollo de una mejor infraestructura, que Angola necesita desesperadamente; integración económica; y lo más importante, paz y seguridad; así como el logro de la estabilidad política y el crecimiento económico. Por lo tanto, la alianza entre los dos países es estratégicamente significativa y deberá estar fundamentada en relaciones de amistad y la cooperación (Hamutenya, 2014).

Ahora bien, el retraso de la paz en Angola durante casi doce años después de la independencia de Namibia en el año de 1990, también postergó la formalización de las relaciones entre los dos países a nivel diplomático, económico y social. No obstante, en septiembre de 1990, se accionó la toma de medidas para establecer una comisión mixta de cooperación, la Joint Commission of Cooperation (JCC) con la finalidad de analizar la consideración de áreas de interés económico para ambos países. Dicha comisión, sentó las bases y promovió instrumentos que luego se tradujeron en acuerdos conjuntos sobre aspectos comerciales y económicos; su amplio enfoque y perspectiva de desarrollo, también significó el establecimiento de una cooperación bilateral formal en áreas asociadas al turismo y la energía (Hamutenya, 2014).

Sin embargo, debido a una serie de factores, Hamutenya (2014) señala que llevó mucho tiempo concluir y firmar acuerdos bilaterales posteriores a la conformación de la JCC; entre éstos, se incluyen los siguientes:

- Angola estaba saliendo de una guerra larga y devastadora y, por lo tanto, enfrentaba sus propias prioridades y desafíos competitivos.

- Después del restablecimiento de la paz en Angola en el año 2002, los retos económicos y de diversa índole en el país exigieron la atención urgente, dejando poco tiempo para considerar la menor presión bilateral infringida por los acuerdos económicos

- La falta de infraestructura, así como las deficiencias de las telecomunicaciones en Angola, causó retrasos en la comunicación.

- Finalmente, la barrera del idioma y otros aspectos técnicos desempeñaron un papel en la prolongación de la conclusión de acuerdos bilaterales.

A pesar de esas deficiencias, en mayo de 2003, Namibia y Angola materializaron un Acuerdo sobre Protección Recíproca y Promoción de Inversiones, que fue ratificado en 2005. El acuerdo buscaba proteger las inversiones y crear condiciones favorables para las empresas en ambos países. En 
marzo de 2004, los dos Estados firmaron un Acuerdo sobre Comercio y Cooperación Económica, también ratificado en 2005. El Centro de Inversiones de Namibia y la Agencia Nacional de Angola para la inversión, estableció un Acuerdo de Cooperación Conjunta en 2008, el cual pretendía promover la generación de estrategias de inversión para implementar programas y proyectos conjuntos. En el año 2009, Namibia y Angola también firmaron un Acuerdo de Asociación Económica que fomentaba y facilitaba el comercio bilateral transfronterizo. Además, para el 2014, Namibia y Angola trabajaran en un Memorando de Entendimiento (MOU) sobre la promoción de desarrollo para pequeñas y medianas empresas (Hamutenya, 2014).

Otros marcos de cooperación entre Angola y Namibia, incluyen entre los más representativos (Hamutenya, 2014):

- La Comisión de la Corriente de Benguela, órgano tripartito que representa Angola, Namibia y Sudáfrica. Es una iniciativa multisectorial intergubernamental destinada a promover la gestión integrada, el desarrollo sostenible y la protección de la corriente de Benguela, gran ecosistema marino, con una rica biodiversidad y de los más productivos del mundo, vulnerable a la destrucción, debido a las actividades humanas como la pesca, minería y transporte marítimo (Small y Gaspar, 2018).

- El tren de Olubido y el Corredor Trans-Cunene: en 1997, Namibia y Angola acordaron formalmente mejorar el sistema de transporte en el Corredor Trans-Cunene mediante la construcción del ferrocarril Olubindo. Representa el principal medio de intercambio de importaciones entre ambas naciones y de mercancías que llegan al puerto de Walvis Bay; es un proyecto financiado por la Unión Europea y el gobierno angoleño; contempla una vía ferroviaria a través de Oshikango-Namibia y Santa Clara-Angola (Tjonneland, 2011), su finalidad es facilitar el transporte en la frontera (Derivet Vidal, 2015).

- Múltiples acuerdos de cooperación hídrica.Dentro de ellos se estableció el Acuerdo para la Creación de la Comisión del Curso del Zambeze (Acuerdo ZAMCOM), firmado, en el 2004, por Angola, Botswana, Malawi, Mozambique, Namibia, Tanzania, Zambia y Zimbabwe; entró en vigor en 2011, para la promoción equitativa del uso de los recursos hídricos e introducir reglas y procedimientos para la resolución de disputas (Wouters, 2013).

- La cuenca del río Okavango: es un acuerdo de cooperación entre Angola, Botswana y Namibia en lo que se refiere a la utilización del río Okavango. Este acuerdo compromete a los Estados miembros a promover el desarrollo coordinado y sostenible de los recursos hídricos (Brachet y Valensuela, 2012). Desde la visión de Edwerd (2009), este tipo de proyectos apunta a aprovechar al máximo los recursos hídricos de los países africanos, en este caso Angola y Namibia, garantizando la protección de los ecosistemas y la gestión de los acuíferos compartidos entre las diferentes naciones del continente.

Sobre este particular, las Naciones Unidas (2018), refiere la existencia de un alto nivel de acuerdos en relación con las cuencas fluviales transfronterizas; así mismo expone que la Comisión de Aguas de la Cuenca del Rio Okavango trabajó con las partes interesadas para diseñar un plan para el uso y conservación de las fuentes hídricas.

- La cuenca del río Kunene: en relación a la gestión de los recursos en esta región, existe también un acuerdo entre los dos Estados. Para Borges (2017), es un proyecto de relevancia estratégica para Angola y Namibia, por cuanto contribuye a mejorar la calidad de vida de la población en la zona fronteriza; el mismo según Tjonneland (2011), se amplía con la presa de Gove; y la Embajada de la República de Angola (2016), refiere que el agua del embalse regará miles de hectáreas para la producción de especies vegetales con el fin de garantizar la seguridad alimentaria de la población. Éste es un compromiso del Banco Mundial para financiar la reconstrucción y expansión de la infraestructura en Angola, con el propósito de propiciar el suministro de agua y electricidad.

El propósito de todos los acuerdos y organizaciones señalados anteriormente, es facilitar el comercio entre los dos países vecinos, a pesar de la existencia de dificultades técnicas y otros 
problemas que han obstaculizado el rápido avance de la integración regional (Steenkamp, 2006; Amado, 2018).

\section{Consideraciones Finales}

El objetivo de este artículo fue desarrollar una revisión teórica sobre el regionalismo en el marco de la economía política, según diversas contextualizaciones que se han planteado sobre éste; tomando el caso de Angola y Namibia como expresión de este proceso en el continente africano; en este orden de ideas, se abordaron conceptualizaciones de región, regionalismo y regionalización, las cuales han sido de especial relevancia para dar explicación y el entendimiento de los procesos de integración regional, específicamente en lo que concierne a la integración entre los mencionados países.

Dentro de este contexto, teóricos como Sánchez Ortiz(2009), Murillo (2004), Bernal Meza (2009), entre otros, exponen que el regionalismo es una forma de integración entre los diferentes estados o naciones que forman parte de una región; en tal sentido, este tipo de procesos implica el desarrollo de convenios de cooperación y colaboración, ayuda mutua para fomentar el desarrollo social, económico y cultural de las partes involucradas.

No obstante, desde la mirada de autores como Haesbaert (2010), resulta particularmente difícil y complicada, la consolidación de un proceso integracionista desvinculado del trasfondo de una región y de un elevado grado de regionalismo y regionalidad ${ }^{8}$.

En materia de economía política, el regionalismo se inserta en el área de las Relaciones Internacionales, ella toma en cuenta los esfuerzos de los estados, en este caso, los del continente africano y dentro de ellos Angola y Namibia, para solventar situaciones acerca dehermandad, democracia y derechos humanos; sus intereses de desarrollo económico y promoción de las fuerzas productivas internas, así también las potencialidades internas y externas de cada nación.

En efecto, en el mundo contemporáneo, los Estados pretenden involucrarse en procesos de integración regional con sus vecinos cercanos, aludiendo a intereses comunes referidos al incremento considerable de su competitividad y su participación en los escenarios internacionales, así como la maximización del aprovechamiento de las ventajas competitivas con la finalidad de atraer mejores y mayores inversiones; en referencia a este aspecto, la proximidad geográfica, así como las similitudes culturales y las preferencias de los consumidores regionales, desempeñan un rol significativo. Por último, otro interés común, radica en la búsqueda de propiciar las condiciones económicas que favorezcan la elevación de la calidad de vida de la población, a través de la generación de mayor cantidad de empleos, el libre tránsito de capitales, bienes y servicios, y en algunos casos, de personas.

De este modo, Namibia y Angola han establecido acuerdos de cooperación orientados a lograr la integración binacional, dentro de ellos está el Acuerdo sobre Protección Recíproca y Promoción de Inversiones, ratificado en 2005, su fin es proteger las inversiones y crear condiciones favorables para las empresas en ambos países. También, el Acuerdo sobre Comercio y Cooperación Económica, en 2005, el Acuerdo de Cooperación Conjunta en 2008; en 2009, Namibia y Angola también firmaron un Acuerdo de Asociación Económica; en 2014, ambas naciones abordaron un Memorando de Entendimiento (MOU) sobre la promoción de desarrollo para pequeñas y medianas empresas.

Otros marcos de cooperación entre Angola y Namibia son: La Comisión de la Corriente de Benguela, el tren de Olubido y el Corredor Trans-Cunene, Acuerdo para la Creación de la Comisión del Curso del Zambeze (Acuerdo ZAMCOM), firmado, en el 2004,Acuerdo sobre la cuenca del río Okavango; el acuerdo acerca de la cuenca del río Kunene, entre otros, dirigidos al mejoramiento de las relaciones, la promoción del desarrollo. Todos ellos en el marco de la integración regional del continente, considerada como una herramienta y estrategia para la estabilización y el desarrollo, incluso, desde la época colonial. En efecto, desde la perspectiva

\footnotetext{
${ }^{8}$ Según Haesbaert (2010), la regionalidad estaría relacionada, de forma genérica, a la propiedad o cualidad de "ser" regional, involucrando con ello la creación concomitante de la "realidad", así como de las representaciones regionales. 
nacional, la integración regional ha sido percibida como un factor de gran relevancia, en función del compromiso que las naciones tienen con el proceso para lograr políticas y acciones como: incremento de la producción, como resultado de la competencia y la especialización; la mejora de la eficiencia, por medio de la competitividad; mayor acceso a los recursos; ampliación y expansión del mercado, fruto del incremento del poder adquisitivo de los compradores; estabilidad política; así como el incremento del poder colectivo de negociación de la región frente al sistema internacional.

En general, el tratamiento teórico explicativo acerca del regionalismo en diversos contextos mundiales, todavía necesita un mayor desarrollo. Desde el final de la Segunda Guerra Mundial, en 1945, y más aún, después de la culminación de la Guerra Fría, a partir de 1989, se ha realizado un significativo esfuerzo académico en relación a la aproximación de la definición consensuada de las regiones y del regionalismo.

Sin embargo, esta voluntad intelectual no se ha traducido en los logros esperados y en la actualidad, los términos de región, regionalismo e integración, permanecen como términos muy ambiguos. Específicamente aludiendo al concepto de región, es perceptible que éste puede ser visto como el elemento que subyace en la comprensión de lo que serían los procesos de regionalización y regionalismo; aun así, tampoco existe un acuerdo en la literatura de las relaciones internacionales sobre la su conceptualización e interpretación unánime.

\section{Referencias Bibliográficas}

Amado, Solange. (2018). Los procesos de integración y cooperación en África. El papel de Angola. Tesis de Doctorado. Tesis de Doctorado en Geografía e Historia. Universidad Complutense, España.

Bach, Daniel. (1998). Régionalisation, mondialisation et fragmentation en Afrique subsaharienne. Karthala, Francia.

Bartesaghi, Ignacio y Pereira, María Eugenia. (2015). Los procesos de integración en África: desarrollo en contextos adversos. Revista PUCE101. Pontificia Universidad Católica, $\begin{array}{llllll}\text { Ecuador } & \text { (pp. } & 2 & - & 29) . & \text { Extraído }\end{array}$ dehttps://ucu.edu.uy/sites/default/files/facultad/fce/dnii/Los procesos de integracion e n Africa.pdf

Benavides de La Vega, Lourdes. (2011). Integración y desarrollo en África. Pensamiento crítico. Extraído dehttp://www.pensamientocritico.org/louben0611.htm

Bernal Meza, Raúl. (2009). El Regionalismo: Conceptos, paradigmas y procesos en el Sistema Mundial Contemporáneo. Aportes para la Integración Latinoamericana. Año XV, $\mathrm{N}^{\mathrm{o}} 21$, Argentina (pp. 12-15). Extraído de https://revistas.unlp.edu.ar/aportes/article/view/3374

Borges, Joao Baptista. (2017). Angola: Delegação interministerial trabalha no Cunene. Agência de Imprensa de Angola. Extraído de https://www.angop.ao/noticiaso/?v link=https://www.angop.ao/angola/pt pt/noticias/economia/2017/0/3/AngolaDelegacao-interministerial-trabalha-Cunene,b313dcf2-f838-4242-94d5-b40b9e991321.html

Brachet, Christophe y Valensuela, Daniel. (2012). Manual para la gestión integrada de los recursos hídricos de las cuencas transfronterizas de ríos, lagos y acuíferos. Red Internacional de Organismos de Cuenca (RIOC) y la Asociación Mundial para el Agua (GWP). Extraído de http://www.repo.funde.org/851/1/Manual-aguas.pdf

Derivet Vidal, Fidel. (2015). La integración económica. La SADC y el papel de Angola. Extraído de http://www.isri.cu/sites/default/files/publicaciones/articulos/boletin0315 0.pdf

Diccionario del Poder Mundial. (2018). Comunidad Internacional. Extraído dehttp://podermundial.net/termino/comunidad-internacional/

Edwerd, Mickel. (2009). El desarrollo de un continente. Boletín del OIEA, 51 - 1. Extraído de https://www.iaea.org/sites/default/files/51102365356_es.pdf. Consulta: 20/11/2020.

Embajada de la República de Angola en el Reino de España (2019). "El país". Extraído de http://www.embajadadeangola.com/embajadadeangola-angola.html 
Fernández, Wilson. (1992). Mercosur: economía, política y estrategia en la integración. Fundación de la Cultura Universitaria, Uruguay.

Gobierno de España. (2018). Integración Regional. Ministerio de Asuntos Exteriores, Unión Europea $\quad$ y Cooperación. Extraído de http://www.exteriores.gob.es/Portal/es/PoliticaExteriorCooperacion/Africa/Paginas/Inte gracionRegional.aspx

Grant, Andrew e Söderbaum, Fredrik. (2003). The New Regionalism in Africa. Ashgate Publishing Limited, Inglaterra.

Haesbaert, Rogério. (2005). Da desterritorialização à multiterritorialidade. Boletín Gaucho. No 5, Argentina(pp. 1-15). Extraído dehttps://seer.ufrgs.br/bgg/article/view/38739

Haesbaert, Rogério. (2010). Región, regionalización y regionalidad: cuestiones contemporáneas, Extraído de http://www.ucs.br/etc/revistas/index.php/antares/article/viewFile/416/361

Hamutenya, Hidipo. (2014). Namibia and Angola: Analysis of a symbiotic relationship. Extraído dehttp://www.kas.de/upload/Publikationen/2014/namibias foreign relations/Namibias Foreign Relations hamutenya.pdf.

Hettne, Björn e Söderbaum, Fredrik. (2000). Theorising the Rise of Regionness. New Political Economy. Vol. 3, No5, Suecia (pp. 457-472). Extraído de https://gup.ub.gu.se/file/119539 Iliffe, John. (1998). África: historia de un continente. University Press, Inglaterra.

Kabunda Badi, Mbuyi. (2009). Integración regional en África: obstáculos y alternativas. Nova Africa.Volumen 1, $\mathrm{N}^{\circ}$ 25, País Vasco(pp. 91 -112). Extraído de http://biblioteca.hegoa.ehu.es/registros/author/737.

Dick Lord, Brig Gen.(2008). From fledgling to eagle: the South African Air Force during the Border War. Editorial 30 Degrees South,Sudáfrica.

Mata Diz, Jamile Bergamaschine y Volpini Silva, Carla Ribeiro.(2011). El regionalismo y la integración en el Mercosur. Revista Ética e Filosofía Política. Volumen 2, No 13, Brasil (pp. 90-109). Extraído dehttp://www.ufff.br/eticaefilosofia/files/2011/05/13 2 diz.pdf.

Mballa, Louis Valentín. (2008). El Estado Africano: entre crisis y conflictos. Razón y Palabra. Volumen 13, $\quad \mathrm{N}^{\mathrm{O}}$ 62,México (S/P). Extraído de: http://www.redalyc.org/pdf/1995/199520738006.pdf.

Mellado, Noemí B. (2013). Regionalismo Sudamericano: sus características. Revista de la Secretaría del Tribunal Permanente de Revisión. Año 1, $\mathrm{N}^{o}$ 1, Argentina, (p. 201). Extraído de https://dialnet.unirioja.es/descarga/articulo/5830251.pdf.

Murillo Zamora, Carlos. (2004). Aproximación a los regímenes de integración regional. Revista Electrónica de Estudios Internacionales. $\mathrm{N}^{\mathrm{o}}$ 8, España (pp. 1-31). Extraído de http://www.reei.org/index.php/revista/num8/articulos/aproximacion-regimenesintegracion-regional.

Naciones Unidas. (2018). Avances en la cooperación en materia de aguas transfonterizas. ONU, Francia.

Oyarzún Serrano, Lorena. (2008). Sobre la naturaleza de la integración regional: teorías y Debates. Revista de Ciencia Política. Volumen 28, No2, 2008, Colombia (p.95-113). Extraído de https://scielo.conicyt.cl/scielo.php? script=sci arttext\&pid=S0718-090X2008000200004.

Pereira, Juan Carlos.(2008). Diccionario de Relaciones Internacionales y Política Exterior. Editorial Ariel, España.

Pipitone, Ugo. (1996). Los nuevos sujetos regionales: raíces históricas y reordenamiento mundial. En Borja Tamayo, Arturo; González González, Guadalupe y Stevenson, Brian J. R, Regionalismo y poder en América: los límites del neorrealismo. CIDE, Miguel Ángel Porrúa, México.

Real Academia Española. (2018). Diccionario de la lengua española. Extraído de http://dle.rae.es/srv/fetch?id=4QWqnmd $\% 7$ C4QbtksL. 
Rodríguez Suárez, Pedro Manuel y Ochoa Bilbao, Luis. (2014). Los regionalismos en el siglo XXI: conceptualización, retos y perspectivas en la nueva estructura internacional. Extraído de http://www.redalyc.org/articulo.oa?id=96031437005.

Sainz, Nora.(2008). Tres modelos de cooperación en América: La Organización de Estados Americanos, el Grupo de Río y el Mercado Común de Sur. Documento de Trabajo, Universidad Autónoma de Barcelona, España.

Sánchez Ortiz, Alfredo. (2009). Globalización y Regionalismo; una perspectiva económico comercial. Extraído de https://ideas.repec.org/a/erv/contri/y2009i2009-0810.html.

Santos, Milton. (1996). Metamorfosis de un espacio habitado. Edit. Oi- kos-Tau, España.

Sepúlveda Ramírez, Leandro. (2001). Construcción regional y desarrollo productivo en la economía de la globalidad. CEPAL, Serie Estudios y Perspectivas, No. 3, Chile.

Small, Lucas y Gaspar, Miklos. (2018). Boletin del OIEA. Extraído de https://www.iaea.org/sites/default/files/publications/magazines/bulletin/bull591/5911213_es.pdf. Consulta: 20/11/2020.

Söderbaum, Fredrik e Shaw, Timothy M. (2003).Theories of New Regionalism. Palgrave Macmillan, Estados Unidos.

Steenkamp, Willem. (2006). Borderstrike!: South Africa into Angola 1975-1980. Just Done Productions, South Africa.

Tjonnland, Elling N. (2011). Desenvolver as infra-estruturas en Amgola. Chr Michelsen Institute, Noruega.

Wouters, Patricia. (2013). Derecho Internacional: Facilitando la cooperación transfronteriza del agua. Elanders, Suecia. 